\section{Verhaltenstherapie}

Praxis $\mid$ Forschung $\mid$ Perspektiven

\title{
Der Placeboeffekt in der antidepressiven Behandlung
}

\author{
Irving Kirsch \\ Harvard Medical School, Beth Israel Deaconess Medical Center, Boston, MA, USA
}

\section{Schlüsselwörter \\ Antidepressiva · Placebo · Placeboeffekt}

\section{Zusammenfassung}

Die vorherrschende Annahme zur Wirkung von Antidepressiva ist, dass diese durch die Behebung eines chemischen Ungleichgewichts, speziell eines Serotoninmangels im Gehirn, zustande kommt. Auswertungen der publizierten und nicht publizierten, d.h. von Pharmaunternehmen zurückgehaltenen Daten zeigen jedoch, dass der meiste (wenn nicht gar komplette) Nutzen durch den Placeboeffekt bewirkt wird. Einige Antidepressiva steigern die Serotoninspiegel, manche senken sie, und andere haben überhaupt keine Auswirkung auf Serotonin. Dennoch zeigen sie alle den gleichen therapeutischen Nutzen. Anstatt Depression zu behandeln, könnten gängige Antidepressiva eine biologische Vulnerabilität verursachen, die das Risiko einer zukünftigen Depression erhöht. Andere Behandlungsarten (z.B. Psychotherapie und körperliche Betätigung) erzeugen den gleichen Kurzzeitnutzen wie Antidepressiva, zeigen bessere Langzeitwirkung und tun dies ohne die Nebenwirkungen und Gesundheitsrisiken der Medikamente.

(c) Irving Kirsch, PhD/S. Karger GmbH, Freiburg

\section{Einführung}

Am 26. Februar 2008 erschien ein Artikel meiner Arbeitsgruppe in der Zeitschrift PLoS Medicine [Kirsch et al., 2008], und als ich an diesem Morgen aufwachte, war dieser Artikel in den Schlagzeilen aller führenden Tageszeitungen in Großbritannien. Einige Monate später wurde ich von Random House, einem großen Buchverlag,

Das englische Original ist online abrufbar unter www.karger.com/doi/10.1159/000443542. Das englische Original wurde in weiten Teilen bereits an anderer Stelle publiziert.

\section{Keywords}

Antidepressants $\cdot$ Placebo $\cdot$ Placebo effect

\section{Summary}

Antidepressants are supposed to work by fixing a chemical imbalance, specifically, a lack of serotonin in the brain. However, analyses of the published and the unpublished data that were hidden by the drug companies reveal that most (if not all) of the benefits are due to the placebo effect. Some antidepressants increase serotonin levels, some decrease serotonin, and some have no effect at all on serotonin. Nevertheless, they all show the same therapeutic benefit. Instead of curing depression, popular antidepressants may induce a biological vulnerability making people more likely to become depressed in the future. Other treatments (e.g., psychotherapy and physical exercise) produce the same short-term benefits as antidepressants, show better long-term effectiveness, and do so without the side effects and health risks of the drugs. dazu eingeladen, aus dem Artikel ein ganzes Buch zu machen (The Emperor's New Drugs: Exploding the Antidepressant Myth [Kirsch, 2009], das seitdem ins Französische, Italienische, Japanische, Polnische und Türkische übersetzt worden ist. 2 Jahre später waren das Buch und die zugrunde liegenden Forschungsergebnisse das Thema eines 5-seitigen Leitartikels in der einflussreichen US-amerikanischen Newsweek. Und nochmals 2 Jahre später war das Buch Gegenstand eines 15-minütigen Berichts in 60 Minutes, dem bekanntesten US-amerikanischen Nachrichtenprogramm. Auf seltsame Weise war ich von einem ungefährlichen Universitätsprofessor

\begin{tabular}{ll}
\hline KARGER & ( Irving Kirsch, PhD/S. Karger GmbH, Freiburg \\
& $1016-6262 / 16 / 0261-0055 \$ 39.50 / 0$ \\
Fax +497614520714 & Accessible online at: \\
Information@Karger.com & www.karger.com/ver \\
www.karger.com &
\end{tabular}


in einen medialen Superhelden - oder Bösewicht, je nachdem wer dazu gefragt wurde - transformiert worden. Aber was genau hatten meine Kollegen und ich getan, um Gegenstand dieser Verwandlung zu werden?

Zur Beantwortung dieser Frage müssen wir ins Jahr 1998 zurückgehen. Damals veröffentlichen Guy Saphirstein - damals noch Student - und ich eine Meta-Analyse über Antidepressiva in einem Online-Journal der American Psychological Association [Kirsch und Sapirstein, 1998]. Zu Beginn unserer Analyse der Daten aus den verfügbaren klinischen Studien waren Sapirstein und ich nicht sonderlich an Antidepressiva, sondern vor allem am Placeboeffeekt interessiert. Der Placeboeffekt hat mich meine ganze wissenschaftliche Karriere hindurch fasziniert und ich habe mich oft gefragt, wie der Glaube, eine Medikation zu erhalten, zu einem Teil der gleichen Effekte führen kann. Wir nahmen damals an, dass Depressionen ein geeigneter Kandidat für die Erforschung von Placeboeffekten sein könnten, da eines ihrer Hauptmerkmale die Hoffnungslosigkeit ist. Wenn man eine depressive Person fragt, was das Schlimmste im Leben sei, dann ist eine sehr häufige Antwort: meine Depressionen. Dieser Sachverhalt wurde von John Teasdale, einem britischen Psychologen, als «Depression wegen Depression» umschrieben, und wenn dies so ist, nahmen wir damals an, dann könnte schon die Hoffnung einer effektiven Behandlung dazu beitragen, die Depression positiv zu beeinflussen. Oder anders gesagt: Die Hoffnung einer Behandlung bringt Hoffnung zum vormals Hoffnungslosen. Etwas in dieser Art hatten wir vor Augen, als wir begannen, den Placeboeffekt bei Antidepressiva zu erforschen.

Sapirstein und ich suchten dann also nach randomisierten Studien, in denen depressive Patienten entweder Placebo oder gar keine Behandlung erhielten. Die Studien, die wir daraufhin fanden, enthielten alle auch einen Antidepressiva-Arm, da - wenig überraschend - nur in solchen Studien auch Placebos verwendet wurden. $\mathrm{Zu}$ diesem Zeitpunkt war ich aber nicht sonderlich an der Wirkung der Antidepressiva interessiert. Ich nahm an, dass diese Medikamente effektiv sind, und ich habe als Psychotherapeut oft Patienten an Psychiater überwiesen, damit diese ihnen Antidepressiva verschreiben. Manchmal bewirkte die dann begonnene Behandlung eine erkennbare Verbesserung, manchmal aber auch nicht. Wenn ein klinischer Effekt bemerkbar wurde, nahm ich an, dass dies auf die Wirkung der Medikation zurückzuführen sei. Auch wenn ich es aufgrund meiner damals schon langjährigen Passion für das Placebo und seine Effekte hätte besser wissen müssen, kam mir damals keine andere Erklärung in den Sinn.

Als wir dann die Daten ausgewertet hatten, waren Sapirstein und ich nicht sonderlich überrascht, dass wir einen substanziellen Placeboeffekt bei Depressionen vorfanden. Was uns aber wirklich überraschte, war, wie klein der spezifische Effekt der Antidepressiva war. 75\% der klinischen Effekte der Antidepressiva-Behandlung wurden nämlich auch bei Depressiven gefunden, die ein Placebo, d.h. eine Pille ohne jeglichen Wirkstoff, erhalten hatten. Es ist wenig überraschend, dass unsere Meta-Analyse sehr umstritten war und hitzige Debatten auslöste. Die Kritiker waren sich einig, dass unsere Ergebnisse nicht korrekt sein konnten und vielleicht auf nicht repräsentativen Studien beruhten - Antidepressiva waren ja schon in vielen Studien untersucht worden und die Wirkung galt entsprechend als gesichert.

Als Antwort auf diese Kritik haben wir uns dann entschlossen, unsere Ergebnisse anhand eines weiteren Datensatzes aus anderen Studien zu replizieren [Kirsch et al., 2002]. Dabei bedienten wir uns einen kleinen Kniffs: Indem wir uns auf den Freedom of Information Act beriefen, konnten wir von der Food and Drug Administration (FDA) die Daten anfordern, die von den pharmazeutischen Unternehmen im Rahmen des Zulassungsverfahrens von 6 gebräuchlichen und häufig verschriebenen Antidepressiva bei der FDA eingereicht worden waren. Die so erhaltenen Daten haben eine Reihe von Vorteilen, wobei die Tatsache, dass die FDA die Daten von allen gesponserten Studien verlangt, d.h. auch von denen, die dann gar nicht publiziert wurden, wohl an erster Stelle zu nennen ist. Wir hatten also nun die Ergebnisse der publizierten und der nicht publizierten Studien. Dies erwies sich im Nachhinein als sehr bedeutsam, da fast die Hälfte aller von den Pharmaunternehmen gesponserten Studien gar nicht veröffentlicht wurde. Nur die Pharmaunternehmen sowie die FDA kannten die Ergebnisse dieser nicht publizierten Studien, und fast alle dieser Studien wiesen keinen signifikanten Unterschied zwischen Placebo und Antidepressivum auf. Ein weiterer Vorteil der FDA-Daten bestand darin, dass alle die gleiche Messgröße - die Hamilton-Skala - zur Erfassung der antidepressiven Wirkung verwendeten, was die Beurteilung der klinischen Bedeutsamkeit der Ergebnisse erleichterte. Und letztendlich waren die FDA-Daten auch die Grundlage für die Zulassung der betreffenden Antidepressiva. Wenn also irgendetwas an diesen Daten suspekt war, dann hätte die Medikation erst gar nicht zugelassen werden dürfen.

In der Analyse der FDA-Daten zeigte sich, dass nur 43\% aller Studien überhaupt einen statistisch signifikanten Unterschied zwischen Antidepressivum und Placebo fanden, die verbleibenden $57 \%$ der Studien zeigten entweder keine oder negative Unterschiede. Unsere Analysen zeigten auch, dass 82\% der Wirkung von Antidepressiva auch nach der Verabreichung von Placebo beobachtet wurden. Wir haben diese Ergebnisse dann nochmals anhand eines weiteren und größeren FDA-Datensatzes repliziert und fanden dabei wieder, dass $82 \%$ der Antidepressiva-Wirkung auch unter Placebo beobachtet werden kann [Kirsch et al., 2008]. Darüber hinaus betrug der Wirkungsunterschied zwischen Placebo und Antidepressivum weniger als 2 Punkte auf der Hamilton-Skala. Die Hamilton-Skala ist ein Fragebogen mit 17 Items und der Gesamtwert liegt zwischen 0 und 53 Punkten, je nachdem wie depressiv die betreffende Person ist. Ein Unterschied von 6 Punkten kann zum Beispiel allein durch eine Veränderung der Schlafgewohnheiten auftreten, ohne jegliche Veränderung der depressiven Symptome. Die 1,8 Punkte Unterschied zwischen Placebo und Medikament waren also sehr klein, beziehungsweise so klein, dass sie als nicht klinisch bedeutsam anzusehen sind, jedenfalls aus Sicht des National Institute for Health and Care Excellence (NICE), das die Behandlungsrichtlinien für den National Health Service (NHS) in Großbritannien herausgibt und einen Unterschied von 3 Punkten als Kriterium für klinische Bedeutsamkeit festgelegt hat [NICE, 2004]. Wenn man also die publizierten und die nicht publizierten 
Daten zusammen betrachtet, zeigt sich kein klinisch bedeutsamer Unterschied zwischen Placebo und antidepressiver Medikation.

Es wurde nun argumentiert, dass das NICE-Kriterium nicht empirisch und damit willkürlich festgelegt wurde [Turner und Rosenthal, 2008], und tatsächlich ist diese Behauptung korrekt. Das 3-Punkte-Kriterium ist genauso zufällig gewählt wie das 5\%-Fehlerniveau in der statistischen Signifikanztestung. Es gibt aber ein nicht zufälliges Kriterium für klinische Signifikanz [Moncrieff und Kirsch, 2015]. Stefan Leucht und Mitarbeiter veröffentlichten 2013 einen Vergleich zwischen der Hamilton-Skala und der Clinical Global Impressions-Improvement (CGI-I)-Skala [Guy, 1976; Leucht et al., 2013]. Die CGI-I-Skala umfasst 7 Items, mit denen ein Behandler die klinische Verbesserung von 1 ( «Zustand ist sehr viel besser») über 4 («Zustand ist unverändert») bis 7 («Zustand ist stark verschlechtert») einschätzen kann. Leucht et al. analysierten insgesamt 7131 Patienten aus 43 Studien und fanden dabei, dass der durchschnittliche Veränderungswert auf der Hamilton-Skala bei den Patienten, die auf der CGI-I-Skala mit «keine Veränderung» eingeschätzt wurden, bei 3 Punkten lag, was genau dem Wert entspricht, der vom NICE als Kriterium für klinisch bedeutsame Veränderung festgesetzt worden war. Es ist also offensichtlich, dass das NICE-Kriterium zu liberal angesetzt ist, da dieser Unterschied aus Sicht des behandelnden Klinikers gar nicht als solcher erkannt wird. Ein sinnvolleres Kriterium wäre ein Unterschied in der Hamilton-Skala, der dann wenigstens aus Sicht des CGI-I zu einer «geringfügigen Veränderung» führen würde. Ein solcher Unterschied lässt sich aus den Ergebnissen der Arbeit von Leucht et al. [2013] auf 7 Hamilton-Punkte beziffern.

An dieser Stelle sei nochmals auf den Unterschied zwischen statistischer und klinischer Signifikanz hingewiesen. Die statistische Signifikanz sagt etwas über die Reliabilität des gefundenen Effekts aus, d.h. ob der Effekt real oder nur ein Zufallsprodukt ist. Über die Größe des Effekts sagt die statistische Signifikanz jedoch nichts aus. Die klinische Signifikanz hingegen nimmt Bezug auf die Effektgröße und darauf, welche Auswirkung diese auf das Leben einer betroffenen Person hat. Nehmen wir als Beispiel eine Studie mit 500000 Personen, in der gefunden wurde, dass Lächeln die Lebenserwartung steigert - um 5 Minuten. Mit einer Studiengröße von $\mathrm{N}=500000 \mathrm{kann}$ ich mehr oder weniger garantieren, dass so etwas signifikant wird, auch wenn das Ergebnis dann klinisch gesehen bedeutungslos ist.

Die Ergebnisse unserer Meta-Analysen wurden seitdem mehrmals repliziert [Fountoulakis und Möller, 2011; Fournier et al., 2010; NICE, 2004; Turner et al., 2008], wobei einige unsere Daten und andere wiederum Daten aus anderen klinischen Studien benutzten. Die FDA hat sogar selbst eine eigene Meta-Analyse über alle von ihr zugelassenen Antidepressiva durchgeführt [Khin et al., 2011]. Und trotz der unterschiedlichen Ansätze zum Umgang mit den Daten sind die Ergebnisse erstaunlich konsistent und liegen immer unter dem Hamilton-Wert, der im CGI-I mit «keine Veränderung» übereinstimmt. Thomas P. Laughren, Direktor der FDAAbteilung für psychiatrische Produkte, sah dies in 60 Minutes ähnlich: «Ich glaube, dass wir alle darin übereinstimmen, das die Unterschiede zwischen Placebo und Antidepressivum in kürzeren Studi- en eher klein sind.» Hier muss aber hinzugefügt werden, dass diese kleinen und klinisch unbedeutsamen Unterschiede zwischen Placebo und Antidepressivum nicht nur in Studien mit kurzer Laufzeit gefunden werden. In einer Meta-Analyse zu publizierten klinischen Studien fand das NICE [2004], dass die Unterschiede zwischen Placebo und Antidepressivum in Studien mit längerer Laufzeit nicht größer waren als in Studien mit kurzer Laufzeit. Der Unterschied zwischen Medikament und Placebo ist klein - so klein, dass nicht einmal Kliniker diesen Unterschied erkennen können.

\section{Depressionsschwere und die Wirksamkeit von Antidepressiva}

Die Kritiker unserer Meta-Analyse von 2002 warfen uns vor, dass unsere Ergebnisse auf Studien mit Patienten basierten, die nicht wirklich depressiv waren. Sie argumentierten, dass bei stärker depressiven Patienten sehr wohl ein bedeutsamer Unterschied gefunden werden könnte. Tatsächlich war dies auch einer der Gründe, warum wir in 2008 die FDA-Daten erneut analysierten [Kirsch et al., 2008]. Dabei kategorisierten wir die klinischen Studien aus der FDA-Datenbank nach dem Depressionsgrad der eingeschlossenen Patienten zu Beginn der Studie entsprechend konventioneller Depressionskategorien. Es zeigte sich, dass tatsächlich nur eine der Studien an nur moderat depressiven Patienten durchgeführt worden war und dass bei dieser Studie mit 0,07 Hamilton-Skalenpunkten kein signifikanter Unterschied zwischen Medikament und Placebo gefunden wurde. Alle anderen Studien waren hingegen an Patienten durchgeführt worden, die zur Baseline eine sehr schwere Depression hatten, und auch dort war der Unterschied zwischen dem Antidepressivum- und dem Placebo-Arm unter dem Wert für klinische Signifikanz.

Dennoch macht der Depressionsgrad einen Unterschied. Patienten mit sehr hohen Werten auf der Hamilton-Skala ( $>28)$ zeigten dabei einen Unterschied zwischen den Antidepressivum- und Placebo-Armen von 4,36 Hamilton-Skalenpunkten, was nach dem NICE-Kriterium als klinisch bedeutsam angesehen werden kann, auch wenn dies natürlich deutlich unter den 7 Hamilton-Skalenpunkten liegt, die einem CGI-I-Rating von «minimaler Verbesserung» entsprechen.

Um herauszufinden, wie viele Patienten denn eigentlich in diese extrem depressive Kategorie fallen, habe ich Mark Zimmermann von der Brown University School of Medicine gebeten, mir die Rohdaten einer Studie zu schicken, in der er und seine Kollegen die Hamilton-Skalenpunkte von ambulanten Patienten mit unipolarer Major Depression erfasst hatten [Zimmerman et al., 2005]. Auf Basis dieser Daten hatten etwa 11\% der Patienten einen HamiltonSkalenwert von 28 oder mehr Punkten. Dies bedeutet im Umkehrschluss, dass fast $90 \%$ der depressiven Patienten nach Verschreibung von Antidepressiva keinen klinischen Nutzen daraus ziehen.

Aber auch diese $11 \%$ können eine Überschätzung der klinischen Bedeutsamkeit darstellen, da Antidepressiva auch solchen Personen verschrieben werden, die gar nicht die Kriterien für eine Major Depression erfüllen. Der Hund meines Nachbarn ist gestorben; 
sein Hausarzt hat ihm ein Antidepressivum verschrieben. Ein Freund hatte Rückenkrämpfe und erhielt dafür Antidepressiva. Ich selbst habe den Überblick darüber verloren, wie viele Personen mir erzählt haben, dass sie Antidepressiva gegen ihre Schlafstörungen erhalten haben, obwohl gerade Schlafstörungen eine der häufigsten Nebenwirkungen von Antidepressiva sind. Ungefähr 20\% aller Patienten mit Schlafstörungen in den USA erhalten Antidepressiva von ihren Hausarzt [Simon und VonKorff, 1997], trotz der Tatsache, dass «die Popularität von Antidepressiva zur Behandlung von Schlafstörungen nicht auf einem Übermaß an überzeugenden Daten, sondern vielmehr auf den Meinungen und Annahmen der behandelnden Hausärzte beruht» [Wiegand, 2008].

Die Suche nach dem Einfluss des initialen Depressionsgrades ergibt kein klares Bild. Einige Forschergruppen fanden den gleichen Zusammenhang wie wir [z.B. Fournier et al., 2010; Khin et al., 2011], andere hingegen nicht [z.B. Fountoulakis et al., 2013; Locher et al., 2015]. Aber alle Meta-Analysen fanden, dass der Unterschied zwischen Antidepressivum und Placebo unter dem NICE-Kriterium für klinische Signifikanz lag. Die Frage, ob Antidepressiva bei der Subpopulation von schwerstdepressiven Patienten wirken oder ob sie bei allen Schweregraden keine klinische Wirksamkeit zeigen, bleibt also weiterhin offen.

\section{Vorhersage des Behandlungseffekts}

Das Ausmaß der Depression ist einer der wenigen Prädiktoren für den Behandlungseffekt, wohingegen die Art der durchgeführten Antidepressiva-Therapie nur einen kleinen bis keinen Effekt hat. Entsprechend wurde in einer Meta-Analyse aus dem Jahr 2011 festgehalten:

"Auf der Grundlage von 234 Studien zeigte sich kein klinisch bedeutsamer Unterschied in der Wirkung oder der Wirksamkeit in Bezug auf die Akut-, Kontinuierungs- und Aufrechterhaltungsbehandlung bei Major Depression. Es zeigten sich weiterhin keine Wirkungsunterschiede in Bezug auf zusätzliche Beschwerden oder Alter, Geschlecht, ethnische Zugehörigkeit und Komorbidität. ... Aufgrund der aktuellen Evidenz zur differenziellen Wirksamkeit kann kein bestimmtes Antidepressivum der zweiten Generation empfohlen werden» [Gartlehner et al., 2011].

Auch wenn der Medikationstyp keinen klinisch bedeutsamen Unterschied im Behandlungsergebnis ausmacht, lässt sich das nicht auf das Placebo übertragen. Fast alle Antidepressiva-Vergleichsstudien beinhalten eine sogenannte «Run-in»-Phase. Hierbei erhalten alle Patienten vor Beginn der eigentlichen Studie für 1-2 Wochen ein Placebo. Nach diesem Run-in werden die Patienten, die eine substanzielle Verbesserung unter Placebo erfahren haben, aus der Studie ausgeschlossen. Entsprechend bleiben nur die Patienten übrig, die entweder nicht oder nur wenig von der Placebobehandlung profitiert haben, und nur diese Patienten erhalten dann randomisiert Antidepressiva oder weiterhin Placebo. Interessanterweise sind es dann genau die Patienten, die im Run-in wenigstens eine geringe Besserung unter Placebo erfuhren, die unter Antidepressiva am ehesten eine Verbesserung erfahren, wobei dieser Effekt nicht nur vom Behandler festgestellt wurde, sondern sich auch in neuronalen Funktionsveränderungen zeigte [Hunter et al., 2006; Quitkin et al., 1998].

\section{Wie erhielten diese Medikamente die Zulassung?}

Wie konnten diese Medikamente mit einer solch geringen Wirksamkeit von der FDA zugelassen werden? Die Antwort ist in den Zulassungskriterien der FDA begründet, die signifikante Unterschiede zwischen Placebo und Medikament in mindestens 2 adäquat durchgeführten Studien fordern. Dabei gibt es aber ein Problem: Es gibt keine Einschränkung der Anzahl an durchgeführten Studien, die benötigt werden, bis man dann 2 signifikante Studien findet. Die Anzahl der negativen Studien, die dabei auftreten, ist dabei nicht von Bedeutung, da hier nur die Signifikanz (und nicht die Nicht-Signifikanz) der Studien zählt.

Der seltsamste Auswuchs dieses Vorgehens der FDA zeigte sich in der Zulassung des Medikaments Viibryd ${ }^{\circledR}$ (Actavis, Inc., Dublin, Irland) im Jahre 2011. Dabei wurden insgesamt 7 placebokontrollierte Studien durchgeführt, wovon die ersten 5 Studien keinerlei signifikante Unterschiede über alle Depressionsparameter und einen mittleren Placebo-Antidepressivum-Unterschied von weniger als 0,5 Hamilton-Skalenpunkten nachweisen konnten. In 2 Studien schnitt sogar das Placebo - wenn auch nicht signifikant - besser ab als das Medikament. Das pharmazeutische Unternehmen führte dann 2 weitere Studien durch und konnte dabei einen kleinen, aber signifikanten Unterschied von 1,7 Hamilton-Skalenpunkten zwischen Placebo und Medikament finden. Der durchschnittliche Unterschied zwischen Placebo und Viibryd über alle 7 Studien lag bei 1,01 Hamilton-Skalenpunkten, was aus Sicht der FDA dann ausreichend für die Zulassung war. Und die von der FDA zugelassene Information an Ärzte und Patienten war: «Die Wirksamkeit von Viibryd ${ }^{\circledR}$ ist in zwei 8-wöchigen, doppelblinden, randomisiert placebokontrollierten Studien nachgewiesen worden.» Die 5 vorangegangenen negativen Studien wurden mit keinem Wort erwähnt.

Dieses Vorgehen war kein Versehen, sondern ist das Abbild einer sorgfältig abgewogenen Taktik der FDA, die schon seit Jahrzehnten Bestand hat. Meines Wissens gibt es nur ein einziges Antidepressivum (Citalopram), bei dem die FDA auch über das Vorliegen negativer Studien informiert. Diese Ausnahme ist auf die Intervention von Paul Leber, dem damaligen Direktor der FDA-Abteilung für neuropharmakologische Medikamente, zurückzuführen, der am 4. Mai 1998 in einem internen Memo schrieb:

«Ein Aspekt bei der Produktinformation bedarf der besonderen Aufmerksamkeit. Der Bericht enthält nicht nur die klinischen Studien, die die antidepressive Wirksamkeit von Citalopram belegen, sondern es werden auch noch adäquate und sauber durchgeführte klinische Studien erwähnt, die diesen Beweis nicht erbringen konnten. Der verantwortliche Direktor ist der Meinung, dass diese Information weder für Patienten noch für verschreibende Ärzte von Belang ist. Ich stimme dieser Meinung nicht zu. Ich glaube, dass der verschreibende Arzt, der Patient und auch derjenige, der für die Kosten schlussendlich aufkommt, wissen soll, auch ohne die offiziellen FDA-Begutachtungsdokumente gelesen haben zu müssen, dass der antidepressive Effekt von Citalopram nicht in allen deswegen durchgeführten Studien gefunden werden konnte. Es ist mir bewusst, dass in klinischen Studien nicht immer der Wirkungsnachweis von eigentlich wirksamen Medikamenten erbracht werden kann, aber ich zweifle daran, dass dies allgemein oder sogar bei allen Medizinern so bekannt ist. Ich bin überzeugt, dass diese nicht nur das Recht haben, davon zu erfahren, sondern ich bin sogar der Meinung, dass sie davon erfahren sollten. Darüber hinaus bin ich 
der Auffassung, dass eine selektive Erwähnung der positiven, bei Nichterwähnung der negativen Studien möglicherweise als «falsch und irreführend s wahrgenommen werden kann.»

Auch wenn ich Paul Leber weder getroffen noch mit ihm korrespondiert habe, möchte ich ihm hiermit für sein mutiges Memo danken - er ist einer meiner Helden.

\section{Der Serotonin-Mythos}

Über die Jahre ist mir etwas Seltsames aufgefallen: Wenn verschiedene Antidepressiva miteinander verglichen werden, sind ihre Effekte immer sehr ähnlich. Als wir in unserer Meta-Analyse von 1998 sahen, wie klein der spezifische Effekt der Medikation war, nahmen wir zuerst an, dass wir hier einen Fehler gemacht hatten. Vielleicht war es falsch, verschiedene Antidepressiva zusammen auszuwerten? Vielleicht hatten wir die Effektivität bestimmter Antidepressiva dadurch unterschätzt, indem wir andere und weniger wirksame Medikamente mit einschlossen? Bevor wir also unsere Meta-Analyse zur Publikation einreichten, untersuchten wir die Daten nochmals auf den Einfluss der verschiedenen Antidepressiva. Einige der untersuchten Medikamente waren selektive Serotonin-Reuptake-Inhibitoren (SSRI), andere waren trizyklische Antidepressiva, und eine dritte Kategorie war weder noch und wurde unter dem Begriff «andere Antidepressiva» zusammengefasst. Und dann gab es da noch eine vierte Kategorie von Medikamenten, die ursprünglich gar nicht als Antidepressiva intendiert waren, aber auch auf ihren Effekt zur Behandlung von Depressionen untersucht wurden, wie beispielsweise Beruhigungsmittel oder Schilddrüsen-Medikamente.

Das erstaunliche Ergebnis dieser Analyse war, dass es nicht darauf ankam, welches Antidepressivum ein Patient erhielt. Der Medikamenteneffekt war immer gleich und 75\% davon konnte auch im Placebo-Arm beobachtet werden. Ich kann mich noch gut daran erinnern, dass mich das damals erstaunte, aber seitdem habe ich dieses Phänomen wiederholt beobachtet. Beispielsweise wurden in der sogenannten $\mathrm{STAR}^{\star} \mathrm{D}$-Studie - mit 35 Millionen US-Dollar die bislang teuerste klinische Antidepressiva-Studie - Patienten, die nicht auf ein SSRI ansprachen, auf ein anderes Antidepressivum gewechselt. Ein Teil der Patienten erhielt dabei einen selektiven Noradrenalin-Reuptake-Inhibitor (SNRI), der dann die Verfügbarkeit von Noradrenalin und Serotonin erhöhen sollte. Weitere Patienten erhielten ein Medikament (NDRI), das Noradrenalin und Dopamin, nicht aber Serotonin erhöhen sollte, und einige Patienten erhielten einfach ein anderes SSRI. Ungefähr jeder vierte Patient zeigte eine klinische Reaktion auf diesen Medikamentenwechsel und die Effekte über die verschiedenen Kategorien hinweg lagen zwischen $26 \%$ und 28\%. Dabei war es aber egal, welches neue Medikament der Patient dabei erhielt, alle wirkten gleich gut.

Die am häufigsten verschriebenen Antidepressiva sind SSRI, die also selektiv auf den Neurotransmitter Serotonin abzielen. Es gibt jedoch auch ein anderes Antidepressivum mit einem ganz anderen Wirkmechanismus. Es heißt Tianeptin und ist in Frankreich als Antidepressivum zugelassen. Tianeptin ist ein selektiver Seroto-
nin-Reuptake-Verstärker/Enhancer (SSRE). Anstatt also die Verfügbarkeit von Serotonin im Gehirn zu erhöhen, soll dieses Antidepressivum dessen Verfügbarkeit verringern. Wenn also die Theorie, dass Depressionen durch ein Zuwenig an Serotonin verursacht werden, korrekt wäre, dann sollten diese SSRE die Depression verstärken. Dem ist aber nicht so - in klinischen Vergleichsstudien zwischen Tianeptin, SSRI und trizyklischen Antidepressiva zeigen 63\% der Patienten eine klinische Verbesserung, verstanden als eine 50\%ige Symptomreduktion, unter diesem SSRE, was auch der Verbesserungsrate unter SSRI, NDRI und Trizyklika in vergleichbaren Studien entspricht. Es macht also keinen Unterschied, welches Medikament verabreicht wird - es kann die Verfügbarkeit von Serotonin erhöhen oder erniedrigen oder auch gar keinen Effekt auf Serotonin haben - der antidepressive Effekt ist der gleiche. Wie aber nennt man Interventionen, deren Effekt unabhängig von ihren chemischen Bestandteilen ist? Ich nenne sie Placebos.

\section{Antidepressiva als aktive Placebos}

Wie gesehen, sind alle Antidepressiva gleich wirksam, und auch wenn die Unterschiede zwischen Antidepressivum und Placebo nicht klinisch bedeutsam sind, sind sie doch statistisch signifikant. Das führt zur naheliegenden Frage: Was ist diesen Medikamenten gemein?

Eine Gemeinsamkeit dieser verschiedenen Antidepressiva sind ihre Nebenwirkungen. Warum soll das aber von Bedeutung sein? Stellen Sie sich vor, Sie seien Teilnehmer in einer klinischen Studie. Sie werden dabei darüber informiert, dass die Studie doppelblind ist und dass sie möglicherweise ein Placebo erhalten. Es wird Ihnen auch mitgeteilt, was die Nebenwirkungen der Medikation sind. Es wird Ihnen außerdem mitgeteilt, dass die therapeutische Wirkung der Medikation erst nach Wochen eintritt, die Nebenwirkungen aber schon eher auftreten. Würden Sie sich nicht fragen, in welchem Behandlungsarm Sie nun gelandet sind? Und wenn Sie dann bestimmte Nebenwirkungen bei sich beobachten, würden Sie dann nicht annehmen, dass Ihnen das echte Medikament verabreicht wird? In einer Studie erkannten 89\% der depressiven Patienten, dass sie das «echte» Antidepressivum erhalten hatten [Rabkin et al., 1986], und in einer anderen Studie, die Antidepressiva und Benzodiazepine zur Behandlung von Panikstörungen untersuchte, konnten 95\% der betreffenden Patienten korrekt erkennen, dass sie Medikamente erhielten [Margraf et al., 1991].

Und nicht nur die Patienten, sondern auch die behandelnden Ärzte - die dann auch die Symptomverbesserung beurteilen durchschauen die eigentlich «blinde» Zuordnung [Margraf et al., 1991; Rabkin et al., 1986]. Dieses Durchschauen der Randomisierung gelingt den Ärzten noch besser als den Patienten. Patienten können zwar einschätzen, ob sie nun das echte Medikament erhalten, aber noch besser erkennen sie, ob sie im Placebo-Arm sind. Ärzte hingegen erkennen beides gleich gut. Die Beurteilung des klinischen Zustands durch den Arzt ist nun der primäre Endpunkt aller Antidepressiva-Studien, die bei der FDA zur Zulassung eingereicht wurden. Entsprechend ist es auch denkbar, dass die Beurtei- 
lung der klinischen Verbesserung durch den Patienten ein genaueres Urteil über die Unterschiede zwischen Antidepressivum und Placebo ergibt.

Vereinfacht ausgedrückt: Klinische Studien sind nicht wirklich doppelblind. Viele Patienten solcher Studien erkennen aufgrund der erlebten Nebenwirkungen, dass sie das Verum erhalten, und entsprechend erkennen dies auch ihre behandelnden Ärzte, die dann zudem noch das Ausmaß der Depression beurteilen. Man kann sich vorstellen, welche Auswirkungen dies auf die Studienergebnisse hat, aber eigentlich braucht man das nicht - es gibt hierzu Daten. Bret Rutherford und Kollegen von der Columbia University haben dazu die Wirkung von Antidepressiva in Studien mit und ohne Placebo-Arm verglichen [Rutherford et al., 2009]. Der wichtigste Unterschied zwischen diesen beiden Studientypen war, dass in den randomisiert placebokontrollierten Studien sowohl die Patienten als auch die behandelnden Ärzte wussten, dass sie entweder Verum oder Placebo erhielten, wohingegen in den Studien ohne Placebo beide wussten, dass nur Antidepressiva verabreicht wurden. Dieses Wissen führte zu einer deutlichen und signifikanten Steigerung der Wirksamkeit der Antidepressiva. Dieser Befund unterstützt die Annahme, dass der relativ kleine Unterschied zwischen Antidepressivum und Placebo auch durch die Entblindung und die Wahrnehmung der Armzuteilung aufgrund der Nebenwirkungen der Antidepressiva mit beeinflusst wird.

\section{Und jetzt?}

Das Antidepressivum hat eine deutliche klinische Veränderung zur Folge. Aber das trifft auch auf das Placebo zu, wobei dessen therapeutische Wirkung fast genauso groß ist. Das führt natürlich zu einem therapeutischen Dilemma. Der auf das Medikament zurückführbare spezifische Effekt von Antidepressiva - verstanden als Differenz zwischen Antidepressivum und Placebo - ist klein, der Placeboeffekt - verstanden als Differenz zwischen keiner Behandlung und Placebo- hingegen ist groß. Welche klinischen Implikationen haben diese Erkenntnisse?

Eine Möglichkeit wäre das Verschreiben von Placebos, was aber die Täuschung von Patienten beinhalten würde. Dies ist nicht nur ethisch mehr als zweifelhaft, sondern würde auch die Beziehung zwischen Kliniker und Patient - eines der bedeutendsten Werkzeuge, über die der Kliniker verfügt - gefährden. Ein anderer Vorschlag geht dahin, Antidepressiva als aktive Placebos zu nutzen. Hier besteht aber das Problem, dass Antidepressiva nicht ohne Risiken sind, da sie Nebenwirkungen haben. Beispielsweise erleben 70-90\% der Patienten unter SSRI sexuelle Funktionsstörungen [Clayton et al., 2006; Serretti et al., 2009] und häufig auch Gewichtszunahme, Schlafstörungen, Übelkeit und Durchfall. Viele Patienten, die versuchen, ihre Antidepressiva abzusetzen, erleben Entzugssymptome [Rosenbaum et al., 1998]. Antidepressiva wurden auch mit Suizidgedanken sowie mit externalisierendem Ver- halten bei Kindern, Jugendlichen und jungen Erwachsenen in Verbindung gebracht [Molero et al., 2015; Stone, 2014; Stone et al., 2009], und bei älteren Erwachsenen wurde über ein erhöhtes Risiko für Herzinfarkt und Todesfälle jeglicher Ursache berichtet [Andrews et al., 2012]. Die Einnahme von Antidepressiva in der Schwangerschaft erhöht das Risiko für Fehlgeburten und selbst nach termingerechter Geburt zu einer erhöhten Wahrscheinlichkeit für Autismus, Missbildungen und persistierende pulmonale Hypertension [Dolmar et al., 2013]. Dabei ist anzumerken, dass dieses erhöhte Risiko mit der Einnahme von Antidepressiva im ersten Trimester assoziiert ist, d.h. zu einem Zeitpunkt, zu dem sich viele Frauen ihrer Schwangerschaft noch gar nicht bewusst sind.

Die wahrscheinlich überraschendste Komplikation bei der Einnahme von Antidepressiva tritt aber bei allen Altersgruppen und Geschlechtern auf: Antidepressiva führen zu einem erhöhten Risiko, wieder an einer Depression zu erkranken. Die Wahrscheinlichkeit einer erneuten Depression ist nach der Behandlung mit Antidepressiva höher als nach jeder anderen Behandlung, inklusiver einer Behandlung mit Placebos [Andrews et al., 2012; Babyak et al., 2000; Dobson et al., 2008]. Das Ausmaß dieses Rezidivrisikos hängt dabei auch davon ab, wie stark das betreffende Antidepressivum in den Neurotransmitterhaushalt im Gehirn eingreift.

Unter Berücksichtigung dieser gesundheitlichen Risiken sollten Antidepressiva nicht als Behandlung der ersten Wahl eingesetzt werden. Es gibt bessere nichtmedikamentöse Alternativen. In einer eigenen Meta-Analyse untersuchten wir verschiedene Behandlungsoptionen gegen Depressionen, inklusive Antidepressiva, Psychotherapie, die Kombination von Psychotherapie und Antidepressiva sowie alternative Behandlungen wie beispielsweise Akupunktur und körperliche Aktivität [Khan et al., 2012]. Wir fanden keine signifikanten Wirkungsunterschiede zwischen diesen Behandlungsmethoden oder zwischen verschiedenen Psychotherapieansätzen. Wenn also verschiedene Behandlungsoptionen gleich wirksam sind, dann sollte die Behandlungsentscheidung auf der Einschätzung der möglichen Risiken und Schäden basieren. Da Antidepressiva im Vergleich zu den anderen wirksamen Behandlungsarten das größte Risiko aufweisen und die meisten Schäden verursachen, sollten sie nur als letztes Mittel bei extrem schweren Depressionen und nur, falls alle anderen Behandlungsformen versagt haben, eingesetzt werden.

\section{Disclosure Statement}

Der Autor erklärt hiermit, dass keine Interessenskonflikte in Bezug auf das vorliegende Manuskript vorliegen. 


\section{Literatur}

-Andrews PW, Thomson JA, Amstadter A, Neale MC: Primum non nocere: an evolutionary analysis of whether antidepressants do more harm than good. Review. Front Psychol 2012;3:117.

Babyak MA, Blumenthal JA, Herman S, et al.: Exercise treatment for major depression: maintenance of therapeutic benefit at 10 months. Psychosom Med 2000;62: 633-638.

Clayton A, Keller A, McGarvey EL: Burden of phase-specific sexual dysfunction with SSRIs. J Affect Disord 2006;91:27-32.

Dobson KS, Hollon SD, Dimidjian S, et al.: Randomized trial of behavioral activation, cognitive therapy, and antidepressant medication in the prevention of relapse and recurrence in major depression. J Consult Clin Psychol 2008;76:468-477.

Domar AD, Moragianni VA, Ryley DA, Urato AC: The risks of selective serotonin reuptake inhibitor use in infertile women: a review of the impact on fertility, pregnancy, neonatal health and beyond. Hum Reprod 2013;28:160-171.

Fountoulakis KN, Möller HJ: Efficacy of antidepressants: a re-analysis and re-interpretation of the Kirsch data. Int J Neuropsychopharmacol 2011;14:405.

Fountoulakis KN, Veroniki AA, Siamouli M, Moller H: No role for initial severity on the efficacy of antidepressants: results of a multi-meta-analysis. Ann Gen Psychiatry 2013;12:26

Fournier JC, DeRubeis RJ, Hollon SD, et al.: Antidepressant drug effects and depression severity: a patientlevel meta-analysis. JAMA 2010;303:47-53

Guy W: ECDEU Assessment Manual for Psychopharmacology - Revised. Volume DHEW Publ No ADM 76338. Rockville, MD, Department of Health, Education, and Welfare 1976, pp. 218-222.

Gartlehner G, Hansen RA, Morgan LC, et al.: Comparative benefits and harms of second-generation antidepressants for treating major depressive disorder. Ann Intern Med 2011;155:772-785
Hunter AM, Leuchter AF, Morgan ML, Cook IA: Changes in brain function (quantitative EEG cordance) during placebo lead-in and treatment outcomes in clinical trials for major depression. Am J Psychiatry 2006;163: 1426-1432.

Khan A, Faucett J, Lichtenberg P, et al.: A systematic review of comparative efficacy of treatments and controls for depression. PLoS One 2012;7:e41778.

Khin NA, Chen YF, Yang Y, et al.: Exploratory analyses of efficacy data from major depressive disorder trials submitted to the US Food and Drug Administration in support of new drug applications. J Clin Psychiatry 2011;72:464.

Kirsch I: The Emperor's New Drugs: Exploding the Antidepressant Myth. London, The Bodley Head, 2009.

Kirsch I, Deacon BJ, Huedo-Medina TB, et al.: Initial severity and antidepressant benefits: a meta-analysis of data submitted to the Food and Drug Administration. PLoS Med 2008;5:e45.

Kirsch I, Sapirstein G: Listening to Prozac but hearing placebo: a meta-analysis of antidepressant medication. Prevention and Treatment 1998; 1:article 0002a.

Locher C, Kossowsky J, Gaab J, et al.: Moderation of antidepressant and placebo outcomes by baseline severity in late-life depression: a systematic review and metaanalysis. J Affect Disord 2015;181:50-60.

Margraf J, Ehlers A, Roth WT, et al.: How 'blind' are double-blind studies? J Consult Clin Psychol 1991;59:184187.

Molero Y, Lichtenstein P, Zetterqvist J, Gumpert CH, Fazel S: Selective serotonin reuptake inhibitors and violent crime: a cohort study. PLoS Med 2015;12:e1001875.

Moncrieff J, Kirsch I: Empirically derived criteria cast doubt on the clinical significance of antidepressantplacebo differences. Contemp Clin Trials 2015;43:6062.

NICE: Depression: Management of Depression in Primary and Secondary Care. Clinical Practice Guideline No 23, 2004. www.nice.org.uk/guidance/cg23 (Zugriff 24. Mai 2005).
Quitkin FM, McGrath PJ, Stewart JW, et al.: Placebo runin period in studies of depressive disorders: clinical, heuristic and research implications. Br J Psychiatry 1998; 173:242-248.

Rabkin JG, Markowitz JS, Stewart JW, et al.: How blind is blind? Assessment of patient and doctor medication guesses in a placebo-controlled trial of imipramine and phenelzine. Psychiatry Res 1986;19:75-86.

Rosenbaum JF, Fava M, Hoog SL, et al.: Selective serotonin reuptake inhibitor discontinuation syndrome: a randomized clinical trial. Biol Psychiatry 1998;44:77-87.

Rutherford BR, Sneed JR, Roose SP: Does study design influence outcome? The effects of placebo control and treatment duration in antidepressant trials. Psychother Psychosom 2009;78:172-181.

Serretti A, Chiesa A: Treatment-emergent sexual dysfunction related to antidepressants: a meta-analysis. J Clin Psychopharmacol 2009;29:259-266.

Simon GE, VonKorff M: Prevalence, burden, and treatment of insomnia in primary care. Am J Psychiatry 1997;154:1417-1423.

Stone M, Laughren T, Jones ML, et al.: Risk of suicidality in clinical trials of antidepressants in adults: analysis of proprietary data submitted to US Food and Drug Administration. BMJ 2009;339:b2880.

Stone MB: The FDA warning on antidepressants and suicidality - why the controversy? N Engl J Med 2014;371: 1668-1671.

Turner EH, Matthews AM, Linardatos E, et al.: Selective publication of antidepressant trials and its influence on apparent efficacy. N Engl J Med 2008;358:252-260.

Turner EH, Rosenthal R: Efficacy of antidepressants. BMJ 2008;336:516-517.

Wiegand MH: Antidepressants for the treatment of insomnia: a suitable approach? Drugs 2008;68:2411-2417.

Zimmerman M, Chelminski I, Posternak MA: Generalizability of antidepressant efficacy trials: differences between depressed psychiatric outpatients who would or would not qualify for an efficacy trial. Am J Psychiatry 2005;162:1370-1372. 